\title{
Different Approaches for Modelling of Heat Transfer in Non-Equilibrium Reacting Gas Flows
}

\author{
E.V. Kustova and E.A. Nagnibeda \\ Saint Petersburg State University \\ Russia
}

\section{Introduction}

Modelling of heat transfer in non-equilibrium reacting gas flows is very important and promising for many up-to-date practical applications. Thus, calculation of heat fluxes is needed to solve the problem of heat protection for the surfaces of space vehicles entering into planet atmospheres.

In high-temperature and hypersonic flows of gas mixtures, the energy exchange between translational and internal degrees of freedom, chemical reactions, ionization and radiation result in violation of thermodynamic equilibrium. Therefore the non-equilibrium effects become of importance for a correct prediction of gas flow parameters and transport properties. The first attempt to take into account the excitation of internal degrees of freedom in calculations for the transport coefficients was made in 1913 by E. Eucken Eucken (1913), who introduced a phenomenological correction into the formula for the thermal conductivity coefficient. Later on, stricter analysis for the influence of the excitation of internal degrees of freedom of molecules on heat and mass transfer was based on the kinetic theory of gases. Originally, in the papers concerning kinetic theory models for transport properties, mainly minor deviations from the local thermal equilibrium were considered for non-reacting gases Ferziger \& Kaper (1972); Wang Chang \& Uhlenbeck (1951) and for mixtures with chemical reactions Ern \& Giovangigli (1994). In this approach non-equilibrium effects were taken into account in transport equations by introducing supplementary kinetic coefficients: the coefficient of volume viscosity in the expression for the pressure tensor and corrections to the thermal conductivity coefficient in the equation for the total energy flux. Such a description of the real gas effects becomes insufficient under the conditions of finite (not weak) deviations from the equilibrium, in which the energy exchange between some degrees of freedom and some part of chemical reactions proceed simultaneously with the variation of gas-dynamic parameters. In this case characteristic times for gas-dynamic and relaxation processes become comparable, and therefore the equations for macroscopic parameters of the flow should be coupled to the equations of physical-chemical kinetics. The transport coefficients, heat fluxes, diffusion velocities directly depend on non-equilibrium distributions, which may differ substantially from the Boltzmann thermal equilibrium distribution. In this situation, the estimate for the impact of non-equilibrium kinetics on gas-dynamic parameters of a flow 
and its dissipative properties becomes especially important. In recent years, these problems receive much attention, and new results have been obtained in this field on the basis of the generalized Chapman-Enskog method Nagnibeda \& Kustova (2009), see also references in Nagnibeda \& Kustova (2009). The kinetic theory makes it possible to develop mathematical models of a flow under different non-equilibrium conditions, i.e. to obtain closed systems of the non-equilibrium flow equations and to elaborate calculation procedures for transport and relaxation properties.

In the present chapter, on the basis of the kinetic theory developed in Nagnibeda \& Kustova (2009), the mathematical models for calculation of heat transfer in strong non-equilibrium reacting mixtures are proposed for different conditions in a flow.

\section{Theoretical models}

The theoretical models adequately describing physical-chemical kinetics and transport properties in a flow depend on relations between relaxation times of various kinetic processes. Experimental data show the significant difference in relaxation times of various processes.

At the high temperature conditions which are typical just behind the shock front, the equilibrium over the translational and rotational degrees of freedom is established for a substantially shorter time than that of vibrational relaxation and chemical reactions, and therefore the following relation takes place Phys-Chem (2002); Stupochenko et al. (1967):

$$
\tau_{\text {tr }}<\tau_{\text {rot }} \ll \tau_{\text {vibr }}<\tau_{\text {react }} \sim \theta \text {. }
$$

In (1), $\tau_{t r}, \tau_{r o t}, \tau_{v i b r}$ and $\tau_{\text {react }}$ are the the relaxation times for the translational, rotational and vibrational degrees of freedom, and the characteristic time for chemical reactions; $\theta$ is the mean time of the variation of gas-dynamics parameters. In this case for the description of the non-equilibrium flow it is necessary to consider the equations of the state-to-state vibrational and chemical kinetics coupled to the gas dynamic equations. It is the most detailed description of the non-equilibrium flow. Transport properties in the flow depend not only on gas temperature and mixture composition but also on all vibrational level populations of different species Kustova \& Nagnibeda (1998).

More simple models of the flow are based on quasi-stationary multi-temperature or one-temperature vibrational distributions. In the vibrationally excited gas at moderate temperatures, the near-resonant vibrational energy exchanges between molecules of the same chemical species occur much more frequently compared to the non-resonant transitions between different molecules as well as transfers of vibrational energy to the translational and rotational ones and chemical reactions:

$$
\tau_{\text {tr }}<\tau_{\text {rot }}<\tau_{V V_{1}} \ll \tau_{V V_{2}}<\tau_{T R V}<\tau_{\text {react }} \sim \theta .
$$

Here $\tau_{V V_{1}}, \tau_{V V_{2}}, \tau_{T R V}$ are, respectively, the mean times for the $\mathrm{VV}_{1}$ vibrational energy exchange between molecules of the same species, $\mathrm{VV}_{2}$ vibrational transitions between molecules of different species and TRV transitions of the vibrational energy into other modes. Under this condition quasi-stationary (multi-temperature) distributions over the vibrational levels establish due to rapid energy exchanges, and equations for vibrational level populations are reduced to the equations for vibrational temperatures for different chemical species. 
Heat and mass transfer are specified by the gas temperature, molar fractions of species and vibrational temperatures of molecular components Chikhaoui et al. (1997).

For tempered reaction regime, with the chemical reaction rate considerably lower than that for the internal energy relaxation, the following characteristic time relation takes place:

$$
\tau_{\text {tr }}<\tau_{\text {int }} \ll \tau_{\text {react }} \sim \theta,
$$

$\tau_{\text {int }}$ is the mean time for the internal energy relaxation. Under this condition, the non-equilibrium chemical kinetics can be described on the basis of the maintaining thermal equilibrium one-temperature Boltzmann distributions over internal energies of molecular species while transport properties are defined by the gas temperature and molar fractions of mixture components Ern \& Giovangigli (1994); Nagnibeda \& Kustova (2009). The influence of electronic excitation of atoms and molecules on the transport properties under the last condition is also considered in Kustova \& Puzyreva (2009).

Finally, if all relaxation processes and chemical reactions proceed faster than gas-dynamic parameters vary, the relaxation times satisfy the relation:

$$
\tau_{\text {tr }}<\tau_{\text {int }}<\tau_{\text {react }} \ll \theta .
$$

Under this condition, on the time scale $\theta$ a gas flow can be assumed thermally and chemically equilibrium or weakly non-equilibrium (Brokaw (1960); Butler \& Brokaw (1957); Vallander et al. (1977)). In this case the closed system of governing equations of a flow contains only conservation equations, and non-equilibrium effects in a viscous flow manifest themselves mainly in transport coefficients.

In the present contribution, for all these approaches, on the basis of the rigorous kinetic theory methods, we propose the closed sets of governing equations of a flow, expressions for transport and relaxation terms in these equations and formulas for the calculation of transport coefficients. The results of applications of proposed models for particular flows are briefly discussed. The comparison of the results obtained for heat transfer in different approaches behind shock waves, in nozzle flows, in the non-equilibrium boundary layer and in a shock layer near the re-entering body is discussed.

\subsection{State-to-state model}

\subsubsection{Distribution functions and governing equations}

The mathematical models of transport properties in non-equilibrium flows of reacting viscous gas mixtures are based on the first-order solutions of the kinetic equations for distribution functions $f_{c i j}(\mathbf{r}, \mathbf{u}, t)$ over chemical species $c$, vibrational $i$ and rotational $j$ energy levels, particle velocities $\mathbf{u}$, coordinates $\mathbf{r}$ and time $t$. In the case of strong deviations from thermal and chemical equilibrium in a flow, the kinetic processes may be divided for rapid and slow ones and the kinetic equations for $f_{c i j}(\mathbf{r}, \mathbf{u}, t)$ can be written in the form Nagnibeda \& Kustova (2009)

$$
\frac{\partial f_{c i j}}{\partial t}+\mathbf{u}_{c} \cdot \nabla f_{c i j}=\frac{1}{\varepsilon} J_{c i j}^{\mathrm{rap}}+J_{c i j}^{\mathrm{sl}}
$$

where $J_{c i j}^{\mathrm{rap}}, J_{c i j}^{\mathrm{sl}}$ are the collision operators for rapid and slow processes, respectively, the small parameter $\varepsilon$ represents the ratio of the characteristic times for rapid and slow processes $\varepsilon=$ $\tau_{\text {rap }} / \tau_{\text {sl }} \sim \tau_{\text {rap }} / \theta \ll 1$. Under the condition (5), the integral operator for rapid processes 
$J_{c i j}^{\text {rap }}$ describes elastic collisions and rotational energy exchange whereas the operator for slow processes $J_{c i j}^{\mathrm{sl}}$ describes the vibrational energy exchange and chemical reactions:

$$
\begin{aligned}
& J_{c i j}^{\mathrm{rap}}=J_{c i j}^{\mathrm{tr}}+J_{c i j}^{\mathrm{rot}}, \\
& J_{c i j}^{\mathrm{sl}}=J_{c i j}^{\mathrm{vibr}}+J_{c i j}^{\mathrm{react}} .
\end{aligned}
$$

The integral operators (6) are given in Ern \& Giovangigli (1994); Nagnibeda \& Kustova (2009). For the solution of the kinetic equations (5), (6) modification of the Chapman-Enskog method for rapid and slow processes Chikhaoui et al. (1997); Kustova \& Nagnibeda (1998) is used. This method makes it possible to derive governing equations of the flow, expressions for the dissipative and relaxation terms in these equations and algorithms for the calculation of transport and reaction rate coefficients. The solution of the equations (5), (6) is sought as the generalized Chapman-Enskog series in the small parameter $\varepsilon$.

The solution of the kinetic equations in the zero-order approximation

$$
J_{c i j}^{\mathrm{rap}(0)}=0
$$

is specified by the independent collision invariants of the most frequent collisions which define rapid processes. These invariants include the momentum and particle total energy which are conserved at any collision, and additional invariants for the most probable collisions which are given by any value independent of the velocity and rotational level $j$ and depending arbitrarily on the vibrational level $i$ and chemical species $c$. The additional invariants appear due to the fact that vibrational energy exchange and chemical reactions are supposed to be frozen in rapid processes. Based on the above set of the collision invariants, the zero-order distribution function takes the form

$$
f_{c i j}^{(0)}=\left(\frac{m_{c}}{2 \pi k T}\right)^{3 / 2} s_{j}^{c i} \frac{n_{c i}}{Z_{c i}^{\text {rot }}(T)} \exp \left(-\frac{m_{c} c_{c}^{2}}{2 k T}-\frac{\varepsilon_{j}^{c i}}{k T}\right)
$$

Here, $n_{c i}$ is the population of vibrational level $i$ of species $c, \mathbf{c}_{c}=\mathbf{u}_{c}-\mathbf{v}, \mathbf{v}$ is the macroscopic velocity, $\varepsilon_{j}^{c i}$ is the rotational energy of the molecule at $j$-th rotational and $i$-th vibrational levels, $T$ is the gas temperature, $m_{c}$ is the molecular mass, $k$ is the Boltzmann constant, $s_{j}^{c i}$ is the rotational statistical weight, $Z_{c i}^{r o t}$ is the rotational partition function. For the rigid rotator model, $\varepsilon_{j}^{c i}=\varepsilon_{j}^{c}, Z_{c i}^{r o t}=Z_{c}^{r o t}=\frac{8 \pi^{2} I_{c} k T}{\sigma h^{2}}, I_{c}$ is the moment of inertia, $h$ is the Planck constant, $\sigma$ is the symmetry factor.

The distribution functions (8) are specified by the macroscopic gas parameters: $n_{c i}(\mathbf{r}, t)$ $\left(c=1, \ldots, L, i=0,1, \ldots, L_{c}, L\right.$ is the number of chemical species, $L_{c}$ is the number of excited vibrational levels in species $c), T(\mathbf{r}, t)$, and $\mathbf{v}(\mathbf{r}, t)$ which correspond to the set of the collision invariants of rapid processes.

The closed set of equations for the macroscopic parameters $n_{c i}(\mathbf{r}, t), T(\mathbf{r}, t)$, and $\mathbf{v}(\mathbf{r}, t)$ follows from the kinetic equations and includes the conservation equations of momentum and total energy coupled to the relaxation equations of detailed state-to-state vibrational and chemical 
kinetics Nagnibeda \& Kustova (2009):

$$
\begin{aligned}
& \frac{d n_{c i}}{d t}+n_{c i} \nabla \cdot \mathbf{v}+\nabla \cdot\left(n_{c i} \mathbf{V}_{c i}\right)=R_{c i}, \quad c=1, \ldots, L, \quad i=0, \ldots, L_{c}, \\
& \rho \frac{d \mathbf{v}}{d t}+\nabla \cdot \boldsymbol{P}=0, \\
& \rho \frac{d U}{d t}+\nabla \cdot \mathbf{q}+\boldsymbol{P}: \nabla \mathbf{v}=0 .
\end{aligned}
$$

Here $\boldsymbol{P}$ is the pressure tensor, $\mathbf{q}$ is the total energy flux, $\mathbf{V}_{c i}$ are diffusion velocities of molecules at different vibrational states, $U$ is the total energy per unit mass:

$$
\rho U=\frac{3}{2} n k T+\rho E_{\mathrm{rot}}+\sum_{c i} \varepsilon_{i}^{c} n_{c i}+\sum_{c} \varepsilon_{c} n_{c} .
$$

$E_{\text {rot }}$ is the rotational energy per unit mass, $\varepsilon_{i}^{c}$ is the vibrational energy of a molecule of species $c$ at the $i$-th vibrational level, $\varepsilon_{c}$ is the energy of formation of the particle of species $c$.

The source terms in the equations (9) are expressed via the integral operators of slow processes:

$$
R_{c i}=\sum_{j} \int J_{c i j}^{\mathrm{sl}} d \mathbf{u}_{c}=R_{c i}^{\mathrm{vibr}}+R_{c i}^{\mathrm{react}}
$$

and characterize the variation of the vibrational level populations and atomic number densities caused by different vibrational energy exchanges and chemical reactions.

For this approach, the vibrational level populations are included to the set of main macroscopic parameters, and the equations for their calculation are coupled to the equations of gas dynamics. Particles of various chemical species in different vibrational states represent the mixture components, and the corresponding equations contain the diffusion velocities $\mathbf{V}_{c i}$ of molecules at different vibrational states. Thus the diffusion of vibrational energies is the peculiar feature of diffusion processes in the state-to-state approximation.

\subsubsection{Transport properties}

In the zero-order approximation of Chapman-Enskog method

$$
\boldsymbol{P}^{(0)}=n k T \boldsymbol{I}, \quad \mathbf{q}^{(0)}=0, \quad \mathbf{V}_{c i}^{(0)}=0 \quad \forall c, i,
$$

$I$ is the unity tensor.

The set of governing equations in this case describes the detailed state-to-state vibrational and chemical kinetics in an inviscid non-conductive gas mixture flow in the Euler approximation Nagnibeda \& Kustova (2009). Taking into account the first-order approximation makes it possible to consider dissipative properties in a non-equilibrium viscous gas.

The first-order distribution functions can be written in the following structural form Kustova \& Nagnibeda (1998):

$$
f_{c i j}^{(1)}=f_{c i j}^{(0)}\left(-\frac{1}{n} \mathbf{A}_{c i j} \cdot \nabla \ln T-\frac{1}{n} \sum_{d k} \mathbf{D}_{c i j}^{d k} \cdot \mathbf{d}_{d k}-\frac{1}{n} \boldsymbol{B}_{c i j}: \nabla \mathbf{v}-\frac{1}{n} F_{c i j} \nabla \cdot \mathbf{v}-\frac{1}{n} G_{c i j}\right) .
$$


The functions $\mathbf{A}_{c i j}, \mathbf{D}_{c i j}^{d k}, \boldsymbol{B}_{c i j}, F_{c i j}$, and $G_{c i j}$ depend on the peculiar velocity $\mathbf{c}_{c}$ and the flow parameters: temperature $T$, velocity $\mathbf{v}$, and vibrational level populations $n_{c i}$, and satisfy the linear integral equations with linearized operator for rapid processes; $\mathbf{d}_{c i}$ are the diffusive driving forces:

$$
\mathbf{d}_{c i}=\nabla\left(\frac{n_{c i}}{n}\right)+\left(\frac{n_{c i}}{n}-\frac{\rho_{c i}}{\rho}\right) \nabla \ln p .
$$

The transport kinetic theory in the state-to-state approximation was developed, for the first time, in Kustova \& Nagnibeda (1998) and is given also in Nagnibeda \& Kustova (2009). The expressions for the transport terms in the equations (9)-(11) in the first order approximation are derived on the basis of the distribution functions (14).

The viscous stress tensor is described by the expression:

$$
\mathbf{P}=\left(p-p_{\text {rel }}\right) \mathbf{I}-2 \eta \mathbf{S}-\zeta \nabla \cdot \mathbf{v I} .
$$

Here, $p_{\text {rel }}$ is the relaxation pressure, $\eta$ and $\zeta$ are the coefficients of shear and bulk viscosity, $\mathbf{S}$ is the deformation rate tensor. The additional terms connected to the bulk viscosity and relaxation pressure appear in the diagonal terms of the stress tensor in this case due to rapid inelastic TR exchange between the translational and rotational energies. The existence of the relaxation pressure is caused also by slow processes of vibrational and chemical relaxation. If all slow relaxation processes in a system disappear, then $p_{\text {rel }}=0$.

The diffusion velocity of molecular components $c$ at the vibrational level $i$ is specified in the state-to-state approach by the expression Kustova \& Nagnibeda (1998); Nagnibeda \& Kustova (2009):

$$
\mathbf{V}_{c i}=-\sum_{d k} D_{c i d k} \mathbf{d}_{d k}-D_{T c i} \nabla \ln T,
$$

where $D_{\text {cidk }}$ and $D_{T c i}$ are the multi-component diffusion and thermal diffusion coefficients for each chemical and vibrational species.

The total energy flux in the first-order approximation has the form:

$$
\mathbf{q}=-\lambda^{\prime} \nabla T-p \sum_{c i} D_{T c i} \mathbf{d}_{c i}+\sum_{c i}\left(\frac{5}{2} k T+\left\langle\varepsilon^{c i}\right\rangle_{\operatorname{rot}}+\varepsilon_{i}^{c}+\varepsilon_{c}\right) n_{c i} \mathbf{V}_{c i},
$$

where $\lambda_{\text {tr }}+\lambda_{\text {rot }}$ is the thermal conductivity coefficient, $\left\langle\varepsilon^{c i}\right\rangle_{\text {rot }}$ is the mean rotational energy. The coefficients $\lambda_{\text {tr }}$ and $\lambda_{\text {rot }}$ are responsible for the energy transfer associated with the most probable processes which, in the present case, are the elastic collisions and inelastic TR- and RR rotational energy exchanges. In the state-to-state approach, the transport of the vibrational energy is described by the diffusion of vibrationally excited molecules rather than the thermal conductivity. In particular, the diffusion of the vibrational energy is simulated by introducing the independent diffusion coefficients for each vibrational state. It should be noted that all transport coefficients are specified by the cross sections of rapid processes excepting the relaxation pressure depending also on the cross sections of slow processes of vibrational relaxation and chemical reactions.

From the expressions (17), (18), it is seen that the energy flux and diffusion velocities include along with the gradients of temperature and atomic number densities also the gradients of all vibrational level populations with multi-component diffusion coefficients depending on the vibrational levels of colliding molecules. In the state-to-state approach, the transport of 
vibrational energy is associated with diffusion of vibrationally excited molecules rather than with heat conductivity. This constitutes the main feature of the heat transfer and diffusion in the state-to-state approach and the fundamental difference between $\mathbf{V}_{c i}$ and $\mathbf{q}$ and the diffusion velocities and heat flux obtained on the basis of one-temperature, multi-temperature or weakly non-equilibrium approaches.

The transport coefficients in the expressions (16)-(18) can be written in terms of functions $\mathbf{A}_{c i j}$, $\mathbf{D}_{c i j}^{d k}, \boldsymbol{B}_{c i j}, F_{c i j}$, and $G_{c i j}$ :

$$
\begin{gathered}
\eta=\frac{k T}{10}[\boldsymbol{B}, \boldsymbol{B}], \quad \zeta=k T[F, F], \quad p_{\text {rel }}=k T[F, G], \\
D_{\text {cidk }}=\frac{1}{3 n}\left[\mathbf{D}^{c i}, \mathbf{D}^{d k}\right], \quad D_{T c i}=\frac{1}{3 n}\left[\mathbf{D}^{c i}, \mathbf{A}\right], \quad \lambda^{\prime}=\frac{k}{3}[\mathbf{A}, \mathbf{A}]
\end{gathered}
$$

Here $[A, B]$ are the bracket integrals associated with the linearized operator of rapid processes. They were introduced in Nagnibeda \& Kustova (2009) for strongly non-equilibrium reacting mixtures similarly to those defined in Ferziger \& Kaper (1972) for a non-reacting gas mixture under the conditions for weak deviations from the equilibrium.

For the transport coefficients calculation in the state-to-state approximation, the functions $\mathbf{A}_{c i j}, \mathbf{D}_{c i j}^{d k}, \boldsymbol{B}_{c i j}, F_{c i j}$, and $G_{c i j}$ are expanded into the Sonine polynomials in the reduced peculiar velocity and those of Waldmann-Trübenbacher in the dimensionless rotational energy. For the coefficients of these expansions, the linear transport systems are derived in Kustova \& Nagnibeda (1998), Nagnibeda \& Kustova (2009), and the transport coefficients are expressed in terms of the solutions of these systems.

Solving transport linear systems for multi-component mixtures in the state-to-state approximation is a very complicated technical problem because a great number of equations should be considered. A simplified technique for the calculation of the transport coefficients keeping the main advantages of the state-to-state approach, is suggested in Kustova (2001). The assumptions proposed in this paper made it possible to noticeably reduce the number of multi-component diffusion and thermal diffusion coefficients and simplify the expressions for the diffusion velocity and heat flux:

$$
\begin{gathered}
\mathbf{V}_{c i}=-D_{c i c i} \mathbf{d}_{c i}-D_{c c} \sum_{k \neq i} \mathbf{d}_{c k}-\sum_{d \neq c} D_{c d} \mathbf{d}_{d}-D_{T c} \nabla \ln T \\
\mathbf{q}=-\lambda^{\prime} \nabla T-p \sum_{c} D_{T c} \mathbf{d}_{c}+\sum_{c i}\left(\frac{5}{2} k T+\left\langle\varepsilon_{j}^{c i}\right\rangle_{\text {rot }}+\varepsilon_{i}^{c}+\varepsilon_{c}\right) n_{c i} \mathbf{V}_{c i} .
\end{gathered}
$$

Here, $\mathbf{d}_{c}$ is the classical diffusive driving force associated to chemical species rather than to the vibrational level Ferziger \& Kaper (1972). It is important to emphasize that in these formulae, after the simplifications, only the self-diffusion coefficients $D_{c i c i}$ depend explicitly on the vibrational quantum number.

The systems for the calculation of the diffusion, viscosity and thermal conductivity coefficients can be solved using the efficient numerical algorithms elaborated in Ern \& Giovangigli (1994) for the solution of linear algebraic systems, or more traditional techniques used in classical monographs on the kinetic theory Chapman \& Cowling (1970); Ferziger \& Kaper (1972); Hirschfelder et al. (1954). 
The expression for the total energy flux may be presented as a sum of contributions of different processes:

$$
\mathbf{q}=\mathbf{q}^{\mathrm{HC}}+\mathbf{q}^{\mathrm{MD}}+\mathbf{q}^{\mathrm{TD}}+\mathbf{q}^{\mathrm{DVE}},
$$

where $\mathbf{q}^{\mathrm{HC}}, \mathbf{q}^{\mathrm{MD}}, \mathbf{q}^{\mathrm{TD}}$, and $\mathbf{q}^{\mathrm{DVE}}$ are, respectively, energy fluxes associated with the heat conductivity of translational and rotational degrees of freedom, mass diffusion, thermal diffusion, and the transfer of vibrational energy.

Fig. 1 shows the contribution of different transport processes in the mixture $\left(\mathrm{N}_{2}, \mathrm{~N}\right)$ to the heat flux variation behind a shock wave and in a nozzle flow along its axis found in the state-to-state approach.
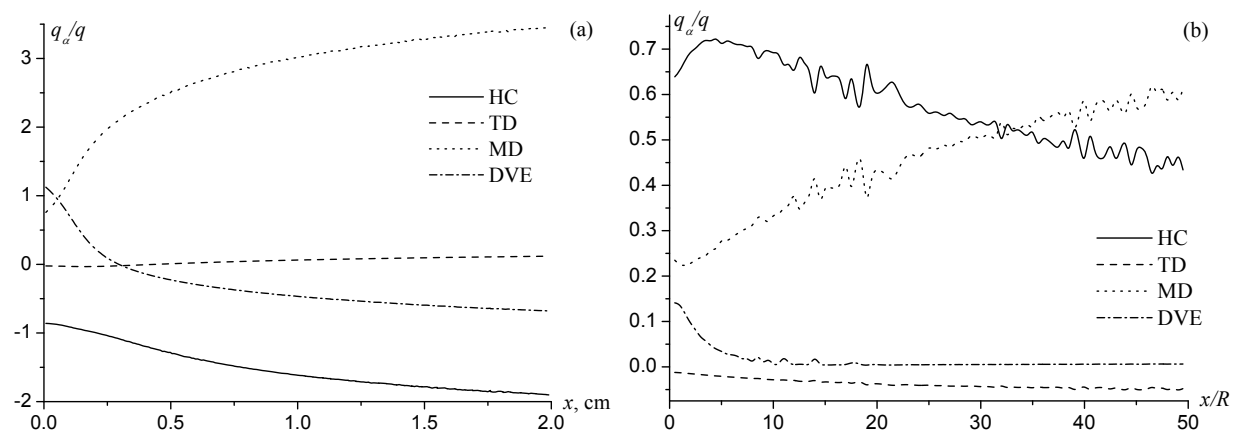

Fig. 1. Ratio of the heat flux due to various processes $(\alpha=$ HC, TD, MD, DVE) to the total heat flux $q$ (a) behind the shock wave $\left(T_{0}=293 \mathrm{~K}, p_{0}=100 \mathrm{~Pa}, M_{0}=15\right)$ as a function of the distance $x$ from the front, and (b) in a conic nozzle $\left(T_{*}=7000 \mathrm{~K}, p_{*}=100 \mathrm{~atm}\right)$ as a function of $x / R$ ( $R$ is the throat radius).

We can see that the contribution of thermal diffusion to the heat flux is small in both flows while mass diffusion of atoms is important in the whole flow region. Diffusion of vibrationally excited molecules plays more important role behind a shock than in a nozzle. Close to the shock front, heat conduction and mass diffusion compensate each other, and the main role in the heat transfer belongs to the diffusion of vibrational states. In an expanding flow, $\mathrm{q}^{\mathrm{DVE}}$ is not negligible only close to the throat (but does not exceed $15 \%$ ).

The model presented in this section gives a principle possibility to take into account the state-to-state transport coefficients in numerical simulations of viscous conducting gas flows under the conditions of strong vibrational and chemical non-equilibrium. The influence of state-to-state vibrational and chemical kinetics on the dissipative processes was studied using this model in the flows of binary mixtures of air components behind shock waves Kustova \& Nagnibeda (1999) and in the nozzle expansion of binary mixtures Kustova, Nagnibeda, Alexandrova \& Chikhaoui (2002) and 5-component air mixture Capitelli et al. (2002). However, even taking into account proposed simplifications of transport coefficients mentioned above, the problem of implementation of the state-to-state model of transport properties in numerical fluid dynamic codes for the flows of multi-component reacting mixtures remains time consuming and numerically expensive for applications particularly if many test cases should be considered. Indeed, the solution of the fluid dynamics equations coupled to the equations of the state-to-state vibrational and chemical kinetics in a flow requires numerical simulation of a great number of equations 
for the vibrational level populations of all molecular species. Moreover, the closed system of macroscopic equations in the state-to-state approach includes the state-dependent rate coefficients of all vibrational energy transitions and chemical reactions. Experimental and theoretical data on these rate coefficients and especially on the cross sections of inelastic processes are rather scanty. Due to the above problems, simpler models based on quasi-stationary vibrational distributions are rather attractive for practical applications.

Such approaches are considered in the next section.

\subsection{Quasi-stationary models}

In quasi-stationary approaches, the vibrational level populations are expressed in terms of a few macroscopic parameters, consequently, non-equilibrium kinetics can be described by a considerably reduced set of governing equations. Commonly used models are based on the Boltzmann distribution with the vibrational temperature different from the gas temperature. However, such a distribution appears not to be justified under the conditions of strong vibrational excitation, since it is valid solely for the harmonic oscillator model, which describes adequately only the low vibrational states. In the present section, the transport kinetic theory is considered on the basis of the non-Boltzmann vibrational distributions taking into account anharmonic vibrations.

\subsubsection{Distribution functions and governing equations}

Quasi-stationary models follow from the kinetic equations (5) under the conditions (2) for the relaxation times. In this case, the integral operator of the most frequent collisions in the kinetic equations (5) for distribution functions includes the operator of $\mathrm{VV}_{1}$ vibrational energy transitions between molecules of the same species along with the operators of elastic collisions and collisions with rotational energy exchanges:

$$
J_{c i j}^{\mathrm{rap}}=J_{c i j}^{\mathrm{tr}}+J_{c i j}^{\mathrm{rot}}+J_{c i j}^{\mathrm{VV}_{1}}
$$

The operator of slow processes $J_{c i j}^{\mathrm{sl}}$ consists of the operator of $\mathrm{VV}_{2}$ vibrational transitions between molecules of different species, the operator describing the transfer of vibrational energy into rotational and translational modes $J_{c i j}^{\mathrm{TRV}}$, as well as the operator of chemical reactions $J_{c i j}^{\text {react: }}$

$$
J_{c i j}^{\mathrm{sl}}=J_{c i j}^{\mathrm{VV}_{1}}+J_{c i j}^{\mathrm{TRV}}+J_{c i j}^{\mathrm{react}} .
$$

For the solution of Eqs. (5) with the collisional operators (24) and (25), the distribution function is expanded into the generalized Chapman-Enskog series in the small parameter $\varepsilon \sim \tau_{V V_{1}} / \theta$. In the zero-order approximation, the following equation for the distribution function is deduced:

$$
J_{c i j}^{\operatorname{tr}(0)}+J_{c i j}^{\mathrm{rot}(0)}+J_{c i j}^{\mathrm{VV}_{1}(0)}=0
$$

The solution of these equations is specified by the invariants of the most frequent collisions. In addition to the momentum and total energy which are conserved in any collision, under the condition (2) there are additional independent invariants of rapid processes: the number of the vibrational quanta in a molecular species $c$, and any value independent of the velocity, vibrational $i$ and rotational $j$ quantum numbers and depending arbitrarily on the particle chemical species $c$. Conservation of vibrational quanta presents an important feature of 
collisions resulting in the $\mathrm{VV}_{1}$ vibrational energy exchange between the molecules of the same species. The existence of a similar invariant for VV transitions in a single-component gas was found for the first time in Treanor et al. (1968) where a non-equilibrium quasi-stationary solution of balance equations for the vibrational level populations was found using this invariant. This solution is now called the Treanor distribution.

In a gas mixture, during $\mathrm{VV}_{1}$ vibrational energy exchange between molecules of the same species, the number of vibrational quanta in a given species keeps constant. The existence of the other additional invariants is explained by the fact that under the condition (2), chemical reactions are supposed to be slow and remain frozen in the most frequent collisions.

Taking into account the system of collision invariants we obtain the zero-order distribution functions:

$$
f_{c i j}^{(0)}=\left(\frac{m_{c}}{2 \pi k T}\right)^{3 / 2} \frac{n_{c} s_{i}^{c}}{Z_{c}^{\text {rot }}(T) Z_{c}^{\text {vibr }}\left(T, T_{1}^{c}\right)} \exp \left(-\frac{m_{c} c_{c}^{2}}{2 k T}-\frac{\varepsilon_{j}^{c}}{k T}-\frac{\varepsilon_{i}^{c}-i \varepsilon_{1}^{c}}{k T}-\frac{i \varepsilon_{1}^{c}}{k T_{1}^{c}}\right) .
$$

Here, $Z_{c}^{\text {vibr }}$ is the non-equilibrium vibrational partition function for molecules of species $c$ :

$$
Z_{c}^{\mathrm{vibr}}=Z_{c}^{\mathrm{vibr}}\left(T, T_{1}^{c}\right)=\sum_{i} s_{i}^{c} \exp \left(-\frac{\varepsilon_{i}^{c}-i \varepsilon_{1}^{c}}{k T}-\frac{i \varepsilon_{1}^{c}}{k T_{1}^{c}}\right) .
$$

$Z_{c}^{\text {rot }}(T)$ is given in Section 2.1, $T_{1}^{c}$ is the temperature of the first vibrational level for each molecular species $c$. It should be noted that vibrational energy $\varepsilon_{i}^{c}$ hereafter is counted from the energy of the zero-th level. The functions (27) represent the local equilibrium Maxwell-Boltzmann distribution of molecules over the velocity and rotational energy levels and the nonequilibrium distribution over the vibrational states and chemical species. For the vibrational level populations, from Eq. (27) it follows:

$$
n_{c i}=\frac{n_{c}}{Z_{c}^{\text {vibr }}\left(T, T_{1}^{c}\right)} s_{i}^{c} \exp \left(-\frac{\varepsilon_{i}^{c}-i \varepsilon_{1}^{c}}{k T}-\frac{i \varepsilon_{1}^{c}}{k T_{1}^{c}}\right) .
$$

It should be emphasized that $n_{c i}$ depend on two temperatures ( $T$ and $T_{1}^{c}$ ) because translational-rotational and vibrational degrees of freedom are not isolated in the most frequent collisions as a consequence of the non-resonant character of $\mathrm{VV}_{1}$ exchange.

The distribution function is specified by the macroscopic parameters $n_{c}, \mathbf{v}, T$, and $T_{1}^{c}$. The temperature $T_{1}^{c}$ is associated to the additional collision invariant $i_{c}$ and is defined by the mean specific number of vibrational quanta $W_{c}$ in molecular species $c$ :

$$
\rho_{c} W_{c}=\sum_{i j} i_{c} \int f_{c i j} d \mathbf{u}_{c}
$$

The expression (29) yields the non-equilibrium quasi-stationary Treanor distribution Treanor et al. (1968) in a multi-component gas mixture. Note that similarly to a single-component gas, the distribution (29) describes adequately only the populations of low vibrational levels $i_{c} \leq i_{c *}$, where $i_{c *}$ corresponds to the minimum of the function $n_{c i}$. It is explained by the fact that the number of vibrational quanta is conserved only at low levels $i_{c} \leq i_{c * *}$. For the collisions of molecules at higher vibrational states $i_{c}>i_{c *}$, the probability of $\mathrm{VV}_{1}$ transitions becomes comparable to that of $\mathrm{VV}_{2}$ and $\mathrm{VT}$ vibrational energy 
exchanges. In the high gas temperature range, if $T \gg T_{1}^{c}$, the level $i_{c *}$ appears to be close to the last vibrational level $i_{c}=L_{c}$ and the Treanor distribution is valid for the entire range of vibrational states. Such a situation is typical for the relaxation zone behind a shock wave. For a strongly excited gas with a high vibrational energy supply $\left(T_{1}^{c} \gg T\right)$, the minimum of the Treanor distribution is located rather low, and the increasing branch of the distribution is not physically consistent. In this case it is necessary to account for various relaxation channels at different groups of vibrational levels. Non-equilibrium vibrational distributions taking into account this effect was obtained for a one-component gas in Gordiets \& Mamedov (1974); Kustova \& Nagnibeda (1997) and are given also in Nagnibeda \& Kustova (2009).

Under the conditions when the anharmonic effects can be neglected, the distribution (29) is reduced to the non-equilibrium Boltzmann distribution with the vibrational temperature of molecular components $T_{\mathrm{v}}^{c}=T_{1}^{c}$ different from $T$ :

$$
n_{c i}=\frac{n_{c}}{Z_{c}^{\mathrm{vibr}}} s_{i}^{c} \exp \left(-\frac{\varepsilon_{i}^{c}}{k T_{1}^{c}}\right),
$$

where the vibrational partition function takes the form

$$
Z_{c}^{\mathrm{vibr}}=Z_{c}^{\mathrm{vibr}}\left(T_{1}^{c}\right)=\sum_{i} s_{i}^{c} \exp \left(-\frac{\varepsilon_{i}^{c}}{k T_{1}^{c}}\right) .
$$

In the case of the local thermal equilibrium, the vibrational temperatures of all molecular species are equal to the gas temperature $T_{1}^{c}=T$, and the Treanor distribution (29) is reduced to the one-temperature Boltzmann distribution:

$$
\begin{gathered}
n_{c i}=\frac{n_{c}}{Z_{c}^{\mathrm{vibr}}} s_{i}^{c} \exp \left(-\frac{\varepsilon_{i}^{c}}{k T}\right), \\
Z_{c}^{\mathrm{vibr}}=Z_{c}^{\mathrm{vibr}}(T)=\sum_{i} s_{i}^{c} \exp \left(-\frac{\varepsilon_{i}^{c}}{k T}\right) .
\end{gathered}
$$

The closed set of governing equations for the macroscopic parameters $n_{\mathcal{c}}(\mathbf{r}, t), \mathbf{v}(\mathbf{r}, t), T(\mathbf{r}, t)$, and $T_{1}^{c}(\mathbf{r}, t)$ were derived in Chikhaoui et al. (2000; 1997), it includes conservation equations for the momentum and the total energy, equations for species number densities and additional relaxation equations for the specific numbers of vibrational quanta $W_{c}$ in each molecular species $c$ :

$$
\begin{aligned}
& \frac{d n_{c}}{d t}+n_{c} \nabla \cdot \mathbf{v}+\nabla \cdot\left(n_{c} \mathbf{V}_{c}\right)=R_{c}^{\text {react }}, \quad c=1, \ldots, L, \\
& \rho \frac{d \mathbf{v}}{d t}+\nabla \cdot \boldsymbol{P}=0, \\
& \rho \frac{d U}{d t}+\nabla \cdot \mathbf{q}+\boldsymbol{P}: \nabla \mathbf{v}=0, \\
& \rho_{c} \frac{d W_{c}}{d t}+\nabla \cdot \mathbf{q}_{\mathrm{w}, c}=R_{c}^{\mathrm{W}}-W_{c} m_{c} R_{c}^{\text {react }}+W_{c} \nabla \cdot\left(\rho_{c} \mathbf{V}_{c}\right), \quad c=1, \ldots, L_{\mathrm{m}} .
\end{aligned}
$$


( $L$ is the total number of species, $L_{\mathrm{m}}$ is the number of molecular species).

The conservation equations for the momentum and total energy (36) and (37) formally coincide with the corresponding equations (10) and (11) obtained in the state-to-state approach. One should however bear in mind that in the multi-temperature approach, the total energy is a function of $T, T_{1}^{c}$, and $n_{c}$ :

$$
\rho U=\frac{3}{2} n k T+\sum_{c=1}^{L_{\mathrm{m}}} \rho_{c} E_{\mathrm{rot}, c}(T)+\sum_{c=1}^{L_{\mathrm{m}}} \rho_{c} E_{\mathrm{vibr}, c}\left(T, T_{1}^{c}\right),
$$

$E_{\mathrm{vibr}, c}\left(T, T_{1}^{c}\right)$ is the specific vibrational energy of species $c$. The transport terms are expressed as functions of the same set of macroscopic parameters.

In Eqs. (35), (38), $\mathbf{V}_{c}$ is the diffusion velocity of species $c$. The value $\mathbf{q}_{\mathrm{w}, c}$ in Eq. (38) has the physical meaning of the vibrational quanta flux of $c$ molecular species and is introduced on the basis of the additional collision invariant of the most frequent collisions $i_{c}$ :

$$
\mathbf{q}_{\mathrm{w}, c}=\sum_{i j} i \int \mathbf{c}_{c} f_{c i j} d \mathbf{u}_{c}
$$

The source terms in Eqs. (35) are determined by the collision operator of chemical reactions

$$
R_{c}^{\text {react }}=\sum_{i j} \int J_{c i j}^{\text {react }} d \mathbf{u}_{c} \text {. }
$$

The production terms in the relaxation equations (38) for the specific numbers of vibrational quanta are expressed as functions of collision operators of all slow processes: $\mathrm{VV}_{2}$ and TRV vibrational energy transfers and chemical reactions,

$$
R_{c}^{\mathrm{w}}=\sum_{i j} i \int J_{c i j}^{\mathrm{sl}} d \mathbf{u}_{c}=R_{c}^{\mathrm{w}, \mathrm{VV}_{2}}+R_{c}^{\mathrm{w}, \mathrm{TRV}}+R_{c}^{\mathrm{w}, \text { react }} .
$$

Thus the equations of non-equilibrium chemical kinetics (35) coupled to the conservation equations of the momentum and the total energy (36), (37), as well as to the relaxation equations (38) for the specific numbers of vibrational quanta in molecular components $W_{c}(38)$ form a closed system of governing equations for the macroscopic parameters of a reacting gas mixture flow in the generalized multi-temperature approach. It is obvious that the system (35)-(38) is considerably simpler than the corresponding system (9)-(11) in the state-to-state approach, as it contains much fewer equations. Indeed, instead of $\sum_{c} L_{c}\left(c=1,2, \ldots, L_{\mathrm{m}}\right.$ stands for the molecular species) equations for the vibrational level populations, one should solve $L_{\mathrm{m}}$ equations for the numbers of quanta and $L_{\mathrm{m}}$ equations for the number densities of the chemical components. Consequently, for a two-component mixture containing nitrogen molecules and atoms, one relaxation equation for $W_{N_{2}}$ and one equation for the number density of $\mathrm{N}_{2}$ molecules should be solved instead of 46 equations for the level populations. While studying the important for practical applications five-component air mixture $\mathrm{N}_{2}, \mathrm{O}_{2}$, $\mathrm{NO}, \mathrm{N}, \mathrm{O}$ in the state-to-state approach, one should solve $L_{\mathrm{N}_{2}}+L_{\mathrm{O}_{2}}+L_{\mathrm{NO}}=114$ equations for the vibrational level populations. In the multi-temperature approach, they are reduced to six equations: three for the molecular number densities and three for $T_{1}^{\mathrm{N}_{2}}, T_{1}^{\mathrm{O}_{2}}$, and $T_{1}^{\mathrm{NO}}$. 
In a system of harmonic oscillators, the relaxation equations for the specific numbers of vibrational quanta $W_{c}(38)$ are transformed into those for the specific vibrational energy:

$$
\rho_{c} \frac{d E_{\mathrm{vibr}, c}}{d t}+\nabla \cdot \mathbf{q}_{\mathrm{vibr}, c}=R_{c}^{\mathrm{vibr}}-E_{\mathrm{vibr}, c} m_{c} R_{c}^{\mathrm{react}}+E_{\mathrm{vibr}, c} \nabla \cdot\left(\rho_{c} \mathbf{V}_{c}\right), \quad c=1,2, \ldots, L_{\mathrm{m}},
$$

with

$$
\mathbf{q}_{\mathrm{vibr}, c}=\varepsilon_{1}^{c} \mathbf{q}_{\mathrm{w}, c,} \quad R_{c}^{\mathrm{vibr}}=\varepsilon_{1}^{c} R_{c}^{\mathrm{w}} .
$$

\subsubsection{Transport properties}

In the zero-order approximation of the Chapman-Enskog method, the transport terms are found taking into account the distribution function (27):

$$
\boldsymbol{P}^{(0)}=n k T \mathbf{I}, \quad \mathbf{q}^{(0)}=0, \quad \mathbf{q}_{\mathrm{w}, \mathrm{c}}^{(0)}=0, \quad \mathbf{V}_{c}^{(0)}=0 \quad \forall c,
$$

and the system (35)-38) takes the form typical for inviscid non-conductive flows of multi-component multi-temperature mixtures.

The first-order distribution functions in the generalized multi-temperature approach take the form (see Chikhaoui et al. (1997); Nagnibeda \& Kustova (2009):

$$
\begin{gathered}
f_{c i j}^{(1)}=f_{c i j}^{(0)}\left(-\frac{1}{n} \mathbf{A}_{c i j} \cdot \nabla \ln T-\frac{1}{n} \sum_{d} \mathbf{A}_{c i j}^{d(1)} \cdot \nabla \ln T_{1}^{d}-\frac{1}{n} \sum_{d} \mathbf{D}_{c i j}^{d} \cdot \mathbf{d}_{d}-\right. \\
\left.-\frac{1}{n} \mathbf{B}_{c i j}: \nabla \mathbf{v}-\frac{1}{n} F_{c i j} \nabla \cdot \mathbf{v}-\frac{1}{n} G_{c i j}\right) .
\end{gathered}
$$

The coefficients $\mathbf{A}_{c i j}, \mathbf{A}_{c i j}^{d(1)}, \boldsymbol{B}_{c i j}, \mathbf{D}_{c i j}^{d}, F_{c i j}$, and $G_{c i j}$ are functions of the peculiar velocity and macroscopic parameters of the particles.

The first-order transport terms in Eqs. (35)-(38) are obtained in Chikhaoui et al. (1997) (see also Nagnibeda \& Kustova (2009)) on the basis of the first-order distribution function (46). The expression for the viscous stress tensor formally coincides with Eq. (16), the shear and bulk viscosity coefficients, as well as the relaxation pressure, are specified in terms of bracket integrals by the formulae (19). However, in the multi-temperature approach, the bracket integrals $[A, B]$ themselves are introduced differently compared to the state-to-state model. In the multi-temperature model the bracket integrals depend on the cross sections of elastic collisions and collisions resulting in the RT and $\mathrm{VV}_{1}$ energy exchanges, i.e. on the cross sections of the most probable processes according to the relation (2) for the characteristic relaxation times.

In the multi-temperature approach, the relaxation pressure $p_{\text {rel }}$ and bulk viscosity coefficient $\zeta$ can be presented as the sums of two terms:

$$
\zeta=\zeta_{\text {rot }}+\zeta_{\text {vibr }}, \quad p_{\text {rel }}=p_{\text {rel }}^{\text {rot }}+p_{\text {rel }}^{\text {vibr }}
$$

where the first term is due to inelastic RT rotational energy exchange, whereas the second is connected to the $V V_{1}$ transitions in each vibrational mode.

The diffusion velocity in the first-order approximation takes the form

$$
\mathbf{V}_{c}=-\sum_{d} D_{c d} \mathbf{d}_{d}-D_{T c} \nabla \ln T
$$


and the diffusion and thermal diffusion coefficients $D_{c d}$ and $D_{T c}$ for the particles of each chemical species are given by the formulae

$$
D_{c d}=\frac{1}{3 n}\left[\mathbf{D}^{c}, \mathbf{D}^{d}\right], \quad D_{T c}=\frac{1}{3 n}\left[\mathbf{D}^{c}, \mathbf{A}\right] .
$$

The total energy flux and the fluxes of vibrational quanta depend on the gradients of the gas temperature $T$, the temperatures of the first vibrational level $T_{1}^{c}$, and the molar fractions of chemical species $n_{c} / n$ :

$$
\begin{gathered}
\mathbf{q}=-\left(\lambda^{\prime}+\sum_{c} \lambda_{\mathrm{vt}, c}\right) \nabla T-\sum_{c}\left(\lambda_{\mathrm{tv}, c}+\lambda_{\mathrm{vv}, c}\right) \nabla T_{1}^{c}-p \sum_{c} D_{T_{c}} \mathbf{d}_{c}+\sum_{c} \rho_{c} h_{c} \mathbf{V}_{c}, \\
\varepsilon_{1}^{c} \mathbf{q}_{\mathrm{w}, c}=-\lambda_{\mathrm{vt}, c} \nabla T-\lambda_{\mathrm{vv}, c} \nabla T_{1}^{c} .
\end{gathered}
$$

The heat conductivity coefficients in the expressions (50), (51) are also introduced on the basis of the bracket integrals:

$$
\begin{array}{cc}
\lambda^{\prime}=\frac{k}{3}[\mathbf{A}, \mathbf{A}], & \lambda_{\mathrm{vt}, c}=\frac{k T_{1}^{c}}{3 T}\left[\mathbf{A}^{c(1)}, \mathbf{A}\right], \\
\lambda_{\mathrm{tv}, c}=\frac{k T}{3 T_{1}^{c}}\left[\mathbf{A}, \mathbf{A}^{c(1)}\right], & \lambda_{\mathrm{vv}, c}=\frac{k}{3}\left[\mathbf{A}^{c(1)}, \mathbf{A}^{c(1)}\right] .
\end{array}
$$

The coefficient $\lambda^{\prime}$ describes the transport of the translational and rotational energy, as well as of a small part of the vibrational energy, which is transferred to the translational mode as a result of the non-resonant $\mathrm{VV}_{1}$ transitions between molecules simulated by anharmonic oscillators. Hence the coefficient $\lambda^{\prime}$ can be represented as a sum of three corresponding terms: $\lambda^{\prime}=\lambda_{\mathrm{tr}}+\lambda_{\mathrm{rot}}+\lambda_{\mathrm{anh}}$. The coefficients $\lambda_{\mathrm{vv}, \mathrm{c}}$ are associated with the transport of vibrational quanta in each molecular species and thus describe the transport of the main part of vibrational energy $\varepsilon_{1}^{c} W_{c}$. The cross coefficients $\lambda_{\mathrm{vt}, c}, \lambda_{\mathrm{tv}, c}$ are specified by both the transport of vibrational quanta and the vibrational energy loss (or gain) as a result of non-resonant $\mathrm{VV}_{1}$ transitions. For low values of the ratio $T_{1}^{c} / T$, the coefficients $\lambda_{\mathrm{anh}}, \lambda_{\mathrm{vt}, c}$, and $\lambda_{\mathrm{tv}, c}$ are much smaller compared to $\lambda_{\mathrm{vv}, c}$, and for the harmonic oscillator model $\lambda_{\mathrm{vt}, c}=\lambda_{\mathrm{tv}, c}=\lambda_{\mathrm{anh}}=0$ since $\mathrm{VV}_{1}$ transitions appear to be strictly resonant. For the same reason, the coefficients $\zeta_{\text {vibr }}$ and $p_{\text {rel }}^{\text {vibr }}$ disappear in a system of harmonic oscillators.

While writing Eq. (50) we take into account the definition for the specific enthalpy of $c$ particles

$$
h_{c}=\frac{5}{2} \frac{k T}{m_{c}}+E_{\mathrm{rot}, c}+E_{\mathrm{vibr}, c}+\frac{\varepsilon_{c}}{m_{c}}, \quad h=\sum_{c} \frac{\rho_{c}}{\rho} h_{c} .
$$

The expressions for the diffusion velocity and heat flux in the multi-temperature approach are significantly different from the corresponding expressions in the state-to-state approach. Within the framework of the state-to-state model, the diffusion velocities and heat flux are determined by the gradients of temperature and all vibrational level populations, in the quasi-stationary approach $\mathbf{V}_{c}$ and $\mathbf{q}$ depend on the gradients of chemical species concentrations, the gas temperature and the temperatures of the first vibrational levels (or, for harmonic oscillators, vibrational temperatures) of molecular species. The number of independent diffusion coefficients in the multi-temperature model is considerably smaller 
than that in the approach accounting for the detailed vibrational kinetics. Therefore the use of the quasi-stationary vibrational distributions noticeably facilitates the heat fluxes calculation in a multi-component reacting gas mixture.

The procedure for the derivation of transport linear systems for the calculation of transport coefficients in the quasi-stationary approaches is similar to that described in Section 2.1.

Following the standard technique, while searching the solutions of the linear integral equations, we expand the unknown functions $\mathbf{A}_{c i j}, \mathbf{A}_{c i j}^{d(1)}, \boldsymbol{B}_{c i j}, \mathbf{D}_{c i j}^{d}, F_{c i j}$, and $G_{c i j}$ specifying the transport coefficients into the series of the Sonine and Waldmann-Trübenbacher polynomials. The trial functions are chosen in accordance with the right-hand sides of the integral equations, which are specified by the zero-order distribution function $f_{c i j}^{(0)}(27)$ which describes the equilibrium distribution over the velocity and rotational energy and non-equilibrium distribution over the vibrational energy. This determines some particular features of the polynomials choice in the present case. Thus, in order to express the dependence of the unknown functions on the internal energy, the Waldmann-Trübenbacher polynomials are introduced for the rotational energy as well as for the part of vibrational energy. The choice of the trial functions proposed in Chikhaoui et al. (1997) (see also Nagnibeda \& Kustova (2009)) makes it possible to derive the linear systems of algebraic equations for expansion coefficients. The transport coefficients are expressed via the solution of these equations.

The coefficients of the transport linear systems depend on the cross sections of elastic collisions and inelastic ones specifying rapid relaxation processes whereas the source terms in governing equations involve the cross sections of slow processes of vibrational relaxation and chemical reactions. An important problem crucial for further development and applications of transport kinetic theory for non-equilibrium reacting gases, is determination of the cross sections of inelastic collisions. The most accurate calculations of the cross sections for inelastic collisions are based on quantum-mechanical and semi-classical trajectory methods Billing \& Fisher (1979); Esposito et al. (2000); Laganà \& Garcia (1994). Among up-to-date analytical models for vibration transition probabilities we can recommend the forced harmonic oscillator model Adamovich \& Rich (1998) and generalizations of the SSH model Armenise et al. (1996); Gordietz \& Zhdanok (1986).

The proposed multi-temperature model was applied in Chikhaoui et al. (2000) for the simulation of gas-dynamic parameters, transport coefficients and heat fluxes in non-equilibrium reacting flows of 5-component air mixture behind shock waves. The flows of binary mixtures of air components behind shock waves are studied in Kustova \& Nagnibeda (1999) and in nozzles in Kustova, Nagnibeda, Alexandrova \& Chikhaoui (2002).

\subsection{One-temperature models}

One-temperature models of heat transfer in reacting flows follow from the kinetic equations under the conditions (3) and (4) when equilibration of translational and internal degrees of freedom proceeds much faster than the variation of gas dynamical parameters. It leads to the local thermal equilibrium in a flow or weak deviations from the local thermal equilibrium. In the case (3), chemical reactions proceed in a strongly non-equilibrium regime on the gas dynamic time scale whereas the condition (4) corresponds to equilibrium or weakly non-equilibrium chemical kinetics. 


\subsubsection{Non-equilibrium reactions regime}

Under the condition (3), the integral operator of rapid processes includes the operators of all collisions resulting in the internal energy variation whereas the integral operator of slow processes is associated only with chemically reactive collisions:

$$
\begin{gathered}
J_{c i j}^{\mathrm{rap}}=J_{c i j}^{\mathrm{tr}}+J_{c i j}^{\mathrm{rot}}+J_{c i j}^{\mathrm{vibr}}=J_{c i j}^{\mathrm{el}}+J_{c i j}^{\mathrm{int}}, \\
J_{c i j}^{\mathrm{sl}}=J_{c i j}^{\mathrm{react}} .
\end{gathered}
$$

The zero-order distribution function takes the form of the thermal equilibrium Maxwell-Boltzmann distribution:

$$
f_{c i j}^{(0)}=\left(\frac{m_{c}}{2 \pi k T}\right)^{3 / 2} \frac{n_{c}}{Z_{c}^{\operatorname{int}}(T)} s_{i j}^{c} \exp \left(-\frac{m_{c} c_{c}^{2}}{2 k T}-\frac{\varepsilon_{i j}^{c}}{k T}\right)
$$

with the gas temperature $\mathrm{T}$,

$$
Z_{c}^{\text {int }}(T)=\sum_{i j} s_{i j}^{c} \exp \left(-\frac{\varepsilon_{i j}^{c}}{k T}\right)
$$

is the internal partition function, $\varepsilon_{i j}^{c}$ is the total internal energy of a particle including rotational and vibrational energies, $n_{c}$ are the non-equilibrium number densities of chemical species. In this approach the vibrational level populations have the form (33).

The governing equations for the macroscopic parameters $n_{c}(\mathbf{r}, t), \mathbf{v}(\mathbf{r}, t)$, and $T(\mathbf{r}, t)$, include the equations of the one-temperature non-equilibrium chemical kinetics coupled to the conservation equations for the momentum and total energy:

$$
\begin{aligned}
& \frac{d n_{c}}{d t}+n_{c} \nabla \cdot \mathbf{v}+\nabla \cdot\left(n_{c} \mathbf{V}_{c}\right)=R_{c}^{\text {react }}, c=1, . ., L, \\
& \rho \frac{d \mathbf{v}}{d t}+\nabla \cdot P=0, \\
& \rho \frac{d U}{d t}+\nabla \cdot \mathbf{q}+\boldsymbol{P}: \nabla \mathbf{v}=0 .
\end{aligned}
$$

Note that the total energy $U$ in Eq. (59) is a function of temperature and species number densities since the vibrational energy is given by

$$
\rho E_{\mathrm{vibr}}=\sum_{c=1}^{L_{\mathrm{m}}} \rho_{c} E_{\mathrm{vibr}, c}=\sum_{c=1}^{L_{\mathrm{m}}} \frac{n_{c}}{Z_{c}^{\mathrm{vibr}}(T)} \sum_{i} s_{i}^{c} \varepsilon_{i}^{c} \exp \left(-\frac{\varepsilon_{i}^{c}}{k T}\right),
$$

where $Z_{c}^{\text {vibr }}(T)$ is given by the formula (34).

The production terms in Eqs. (57) are defined by the operator of reactive collisions.

In the zero-order approximation, the equations (57)-(59) describe an in-viscid non-conductive gas flow of a multi-component mixture with non-equilibrium chemical reactions.

The first order transport terms are found using the procedure similar to one described in the previous sections. 
The viscous stress tensor in the one-temperature approach is again expressed by the formula (16), the diffusion velocity is specified by Eq. (48). The coefficients of shear and bulk viscosity, the relaxation pressure and the diffusion and thermal diffusion coefficients are calculated using Eqs. (19), (49). However, one should bear in mind that in the one-temperature approach, contrary to the multi-temperature and state-to-state models, the bracket integrals in (19) related to the viscosity coefficients and relaxation pressure include the cross sections of elastic and all inelastic non-reactive collisions. The viscosity coefficients are determined by the cross sections of all kinds of energy transitions. The relaxation pressure depends on the same cross sections and, in addition, on the cross sections of chemically reactive collisions. The bracket integrals specifying the diffusion and thermal diffusion coefficients also differ from those in the multi-temperature approximation because they are specified by the cross sections of other collisional processes and also due to the different form of the zero-order distribution functions. The total energy flux in the one-temperature approach is associated with thermal conductivity, diffusion and thermal diffusion:

$$
\mathbf{q}=-\lambda^{\prime} \nabla T-p \sum_{c} D_{T_{c}} \mathbf{d}_{c}+\sum_{c} \rho_{c} h_{c} \mathbf{V}_{c}
$$

the partial thermal conductivity coefficient is defined as follows

$$
\lambda^{\prime}=\frac{k}{3}[\mathbf{A}, \mathbf{A}]
$$

and describes the transport of all kinds of internal energy: $\lambda^{\prime}=\lambda_{\mathrm{tr}}+\lambda_{\text {int }}=\lambda_{\mathrm{tr}}+\lambda_{\text {rot }}+$ $\lambda_{\text {vibr }}\left(\lambda_{\text {vibr }}\right.$ is the vibrational thermal conductivity coefficient). The thermal conductivity coefficient depends on the cross sections of elastic and all inelastic collisions excepting those chemically reactive. It is obvious that in the given case, the heat flux (61) takes the simplest form compared to that found in the framework of the state-to-state and multi-temperature approaches since it is determined only by the gradients of the gas temperature and molar fractions of the chemical species. The thermal conductivity coefficient $\lambda^{\prime}$ cannot be measured experimentally since there is a thermal diffusion phenomenon also related to the temperature gradient. For this reason, instead of the coefficient $\lambda^{\prime}$, another heat conductivity coefficient is often introduced Ferziger \& Kaper (1972)

$$
\lambda=\lambda^{\prime}-n k \sum_{c} k_{T c} D_{T c}
$$

where $k_{T c}$ are the thermal diffusion ratios:

$$
\sum_{d} D_{c d} k_{T d}=D_{T c}, \quad c=1, \ldots, L
$$

In this case, the total energy flux can be rewritten as

$$
\mathbf{q}=-\lambda \nabla T+p \sum_{c}\left[k_{T c}+\left(\frac{5}{2}+\left\langle\frac{\varepsilon_{i j}^{c}}{k T}\right\rangle+\frac{\varepsilon_{c}}{k T}\right) \frac{n_{c}}{n}\right] \mathbf{V}_{c},
$$

$\left\langle\varepsilon_{i j}^{c}\right\rangle$ is the internal energy averaged with the Boltzmann distribution. 
The coefficient $\lambda$ can be measured experimentally in a gas mixture under the steady-state conditions. For a single-component gas, since diffusion and thermal diffusion processes are absent, the coefficients $\lambda$ and $\lambda^{\prime}$ coincide.

The transport algorithms for the one-temperature mixture with non-equilibrium chemical reactions are given Nagnibeda \& Kustova (2009). The mathematical properties of transport linear systems and transport coefficients are discussed in Ern \& Giovangigli (1994).

\subsubsection{Chemical equilibrium regime}

Under the conditions of weak deviations from local thermal and chemical equilibrium, the kinetic equations for distribution functions have the form

$$
\frac{\partial f_{c i j}}{\partial t}+\mathbf{u}_{c} \cdot \nabla f_{c i j}=\frac{1}{\varepsilon} J_{c i j}
$$

where integral operator $J_{c i j}$ describes all kinetic processes including all kinds of energy transitions and chemical reactions, $\varepsilon=\tau / \theta \ll 1, \tau$ is the mean time of kinetic processes.

The kinetic theory of transport properties in weakly non-equilibrium reacting flows is developed in Ern \& Giovangigli (1998); Rydalevskaya (1977; 2003). Zero-order solutions of Eqs. (66) nullify the total colliding operator and are found on the basis of the invariants of all types of collisions. In reacting gas mixtures, the set of collision invariants consists not only of the momentum and total energy (including the energy of formation), but contains also additional invariants related to chemical reactions. Such invariants are represented by the numbers of elements (atoms or other non-separable elements), which either exist in the free state or form molecules: $\psi_{c}^{(\lambda)}=k_{\lambda c}$ is the number of elements of the type $\lambda$ $(\lambda=1, \ldots, \Lambda)$ in a particle of chemical species $c$ (for atomic species, $k_{\lambda \lambda}=1$ ). The zero-order distribution function corresponding to the local thermal and chemical equilibrium can be written in the form (55) where the number densities of chemical species $n_{c}$ have the form Nagnibeda \& Kustova (2009); Rydalevskaya (2003)

$$
n_{c}=\frac{Z_{c}(T)}{V} \exp \left(\sum_{\lambda} \gamma_{\lambda} k_{\lambda c}\right) \exp \left(\frac{D_{c}}{k T}\right),
$$

$V$ is the gas volume, $D_{c}$ is the dissociation energy, and $Z_{\mathcal{C}}(T)$ is the total partition function

$$
Z_{c}=Z_{c}^{\operatorname{tr}} Z_{c}^{\text {int }}
$$

$Z_{c}^{\operatorname{tr}}$ is the translation partition function:

$$
Z_{c}^{\operatorname{tr}}(T)=\left(2 \pi k T m_{c}\right)^{3 / 2} \frac{V}{h^{3}}
$$

$h$ is the Planck constant.

The parameters $\gamma_{\lambda}(\mathbf{r}, t)$ are connected to $\tilde{n}_{\lambda}$, which represent the numbers of atoms $\lambda$ per unit volume:

$$
\sum_{c} k_{\lambda c} n_{c}=\tilde{n}_{\lambda}
$$


Note that the number densities of species $n_{c}$ satisfy the equations of chemical equilibrium

$$
R_{c}^{\text {react }(0)}=0
$$

and can be derived in the form (67) directly from the above equations.

The set of governing equations for the macroscopic parameters $\mathbf{v}(\mathbf{r}, t), T(\mathbf{r}, t)$, and $\widetilde{n}_{\lambda}(\mathbf{r}, t)$ $(\lambda=1, \ldots, L)$ follows from the kinetic equations and contains the conservation equations of momentum, total energy and number densities of atoms (or elements). The first-order transport terms differ from those obtained for the case of strongly non-equilibrium chemical reactions considered above in Section 2.3.1. The stress tensor in the viscous gas approximation can be obtained in the form (16), but in the considered case, $p_{\text {rel }}=0$ since slow kinetic processes are absent. The bulk viscosity coefficient is defined by the cross sections of all inelastic processes including chemical reactions. In Ern \& Giovangigli (1998); Rydalevskaya (1977), the heat flux in the case of weak deviations from the chemical equilibrium is expressed in terms of $\nabla T$, the gradients of macroscopic parameters $\gamma_{\lambda}$ and element diffusion velocities. As is emphasized in Ern \& Giovangigli (1998), it is more convenient to use the heat flux formula written in the conventional form as a function of the gas temperature gradient, species diffusive driving forces and species diffusion velocities. This form of the heat flux is useful for practical calculations and for a comparison with experimentally measured coefficients, as well as for the estimate of the contribution of chemical reactions to the total energy flux.

One should bear in mind the difference between heat fluxes calculated in a chemically non-equilibrium one-temperature gas flow and in the regime of weak deviations from the chemical equilibrium even if the fluxes are expressed in terms of the same macroscopic parameters $n_{c}, p$, and $T$. As a matter of fact, the transport coefficients in the two approaches depend on the cross sections of different processes: while in the case of weak chemical non-equilibrium, the transport coefficients depend on the cross sections of all energy exchanges and chemical reactions, in the one-temperature approach for a strongly chemically non-equilibrium gas, they are specified only by the cross sections of energy exchange. Nevertheless, if one assumes that the cross sections of chemically reactive collisions weakly contribute to the thermal conductivity and multi-component diffusion coefficients, one can derive the expression for $q$ in the regime of weak deviations from the chemical equilibrium from the Eq. (61) obtained in the one-temperature approach, simply substituting chemically equilibrium distributions (67) into the latter formula. Then the thermal conductivity coefficient $\lambda^{\prime}$ can be represented by the expression suggested in the Brokaw (1960); Butler \& Brokaw (1957):

$$
\lambda=\lambda_{\text {tr }}+\lambda_{\text {int }}+\lambda_{\text {react }}
$$

where $\lambda_{\text {react }}$ is associated with the contribution of equilibrium chemical reactions to the thermal conductivity. The coefficient $\lambda_{\text {react }}$ can be easily calculated on the basis of equilibrium distributions.

\section{Results}

In the most of the papers on computational fluid dynamics, for numerical simulations of high-temperature and high-enthalpy reacting flows the heat transfer and transport coefficients 
are described without taking into account non-equilibrium effects. The kinetic theory approach makes it possible to include these effect in a numerical scheme.

In order to evaluate transport properties in particular flows of non-equilibrium reacting mixtures on the basis of the kinetic theory models, it is necessary to solve numerically the system of governing equations for the macroscopic parameters in the first-order approximation of the generalized Chapman-Enskog method. In the frame of the rigorous formalism, the transport coefficients in these equations should be calculated at each step of the numerical solution. Such a technique, even using the simplifications discussed in Section 2.1, appears to be extremely time-consuming particularly in the state-to-state approximation. In Kustova \& Nagnibeda (1999), an approximate approach for evaluation of dissipative properties in non-equilibrium flows was suggested. First, the vibrational level populations (in the state-to-state approach) or vibrational temperatures of molecular species (in the multi-temperature approach), molar fractions of atoms, and gas temperature were found from the governing equations in the zero-order approximation. Then the obtained non-equilibrium distributions were used to calculate the transport coefficients, diffusion velocities, and heat fluxes on the basis of the accurate formulae of the kinetic theory. This approach was used for the evaluation of transport properties in the flows of non-equilibrium reacting mixtures of air components behind shock waves Chikhaoui et al. (2000); Kustova \& Nagnibeda (1999), in the nozzle expansion Capitelli et al. (2002); Kustova, Nagnibeda, Alexandrova \& Chikhaoui (2002), in the hypersonic boundary layer Armenise et al. (2006; 1999); Kustova, Nagnibeda, Armenise \& Capitelli (2002) (see also Nagnibeda \& Kustova (2009)).

Below, we present some results of these applications concerning heat transfer in particular flows studied using the state-to-state, multi-temperature and one-temperature models.

In Fig. 2, the variation of the total energy flux in the relaxation zone behind the shock front in $\mathrm{N}_{2} / \mathrm{N}$ mixture obtained in the state-to-state, two-temperature and one-temperature approximations is given for the following conditions in the free stream: $T_{0}=293 \mathrm{~K}$, $p_{0}=100 \mathrm{~Pa}, M_{0}=15$. Distributions in the free stream are assumed to be equilibrium with the temperature $T_{0}$. Comparing the heat flux values obtained in different approaches, one can notice that the one-temperature and two-temperature approaches substantially underestimate the absolute values for the heat flux in the very beginning of the relaxation zone, where the process of vibrational excitation is essential. Calculations show that in the one-temperature approach, heat conductivity coefficient $\lambda^{\prime}$ noticeably exceeds the thermal conductivity coefficient obtained within the two-temperature and state-to-state models since, in the former case, it includes the coefficient $\lambda_{\text {vibr }}$ associated with the vibrational energy transfer (see sections 2.1, 2.2, 2.3).

Figure 3 presents the evolution of the thermal conductivity coefficient $\lambda^{\prime}$ at the gradient of the gas temperature $T$ in the expression for the total heat flux and the coefficient $\lambda_{\mathrm{v}}=\lambda_{\mathrm{tv}}+\lambda_{\mathrm{vv}}$ appearing at the gradient of the vibrational temperature $T_{1}$ or $T_{\mathrm{V}}$ in the multi-temperature approaches along the nozzle axis in dependence of the dimensionless distance from the reservoir $x / R$ ( $R$ is the throat radius). Two mixtures, $\mathrm{O}_{2} / \mathrm{O}$ and $\mathrm{N}_{2} / \mathrm{N}$ are studied in a conic nozzle with the following initial conditions in the reservoir: $T^{*}=4000 \mathrm{~K}, p^{*}=100 \mathrm{~atm}$ (for $\mathrm{O}_{2} / \mathrm{O}$ ) and $T^{*}=7000 \mathrm{~K}, p^{*}=100 \mathrm{~atm}$ (for $\mathrm{N}_{2} / \mathrm{N}$ ). The coefficients are calculated within four models: the state-to-state approach, two-temperature model for anharmonic and harmonic oscillators, and one-temperature approach. The expressions for the heat flux in different approaches are given by the formulae (18), (50), and (61). The discrepancy between 


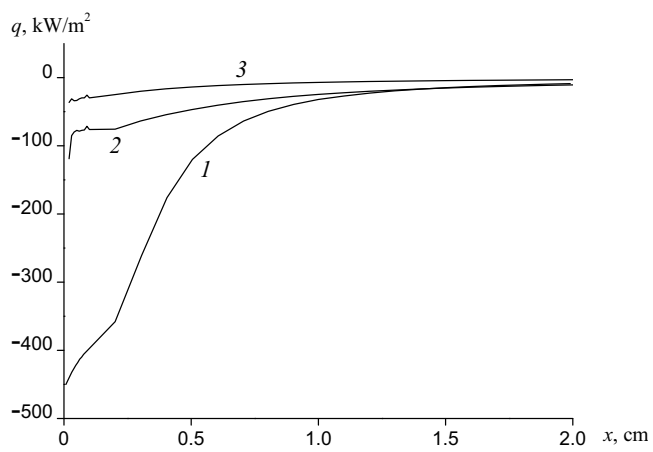

Fig. 2. The heat flux $q$ as a function of $x$. The curves 1, 2, 3 represent the state-to-state, two-temperature, and one-temperature approaches, respectively.

the coefficients obtained within various models appears to be more pronounced in the mixture $\mathrm{N}_{2} / \mathrm{N}$, since in this case the non-equilibrium factor $T_{1} / T$ is sufficiently high (it reaches 8 under the considered conditions), and vibrational excitation and the influence of anharmonicity on the coefficients $\lambda^{\prime}$ and $\lambda_{\mathrm{V}}$ is more important. Contrary to the relaxation zone behind a shock wave, the one-temperature approach results in underestimation for the coefficient $\lambda^{\prime}$, although it includes the coefficient $\lambda_{\text {vibr. }}$. This is a consequence of inadequately low temperature found in this approach.
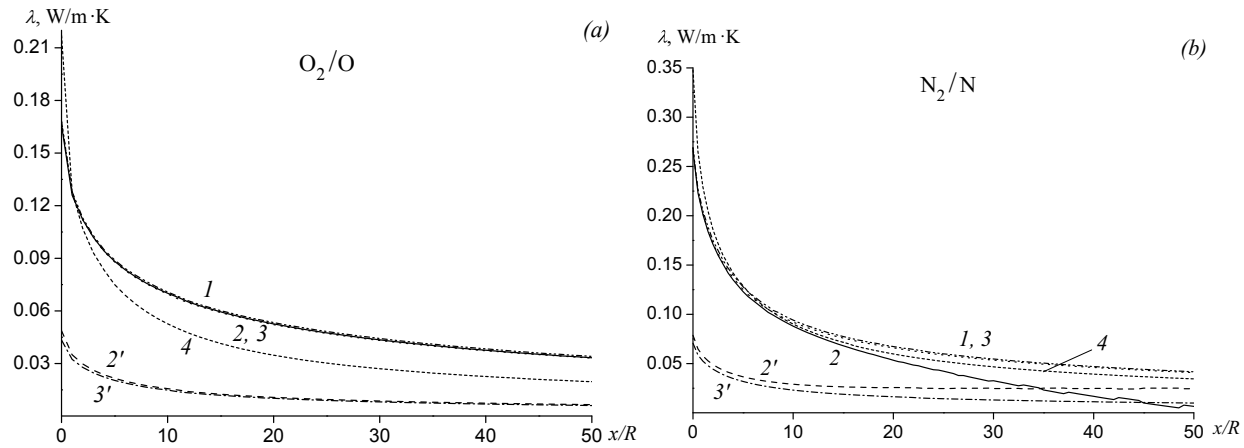

Fig. 3. The thermal conductivity coefficients $\lambda^{\prime}, \lambda_{\mathrm{v}}$ as functions of $x / R$ in different approaches. The curve 1: the coefficient $\lambda^{\prime}$ in the state-to-state approach; $2,2^{\prime}$ : the coefficients $\lambda^{\prime}$ and $\lambda_{\mathrm{V}}$ in the two-temperature approach (anharmonic oscillator); $3,3^{\prime}$ : the coefficients $\lambda^{\prime}$ and $\lambda_{\mathrm{V}}$ in the two-temperature approach (harmonic oscillator); 4 : the coefficient $\lambda^{\prime}$ in the one-temperature approach.

In Fig. 4, the heat flux q calculated for the same flow in the four approaches is presented. The heat flux decreases with $x$, since the gradients of the macroscopic parameters also decrease with the distance from the throat. While the one-temperature model yields underestimated heat flux, the two-temperature quasi-stationary models provide a satisfactory accuracy for $\mathbf{q}$. For the $\mathrm{N}_{2} / \mathrm{N}$ mixture, the maximum deviation of the heat flux found in the non-equilibrium quasi-stationary approaches from that obtained within the most rigorous state-to-state model is $4 \%$ and $10 \%$ for anharmonic and harmonic oscillators, respectively, and, correspondingly, 
$7 \%$ and $10 \%$ for the $\mathrm{O}_{2} / \mathrm{O}$ mixture. It can be seen that non-equilibrium vibrational distributions do not affect essentially the heat flux in a nozzle though distributions themselves found in various approaches differ dramatically. Note that in a flow behind a shock wave the significant influence of a kinetic model on the heat flux values is found.
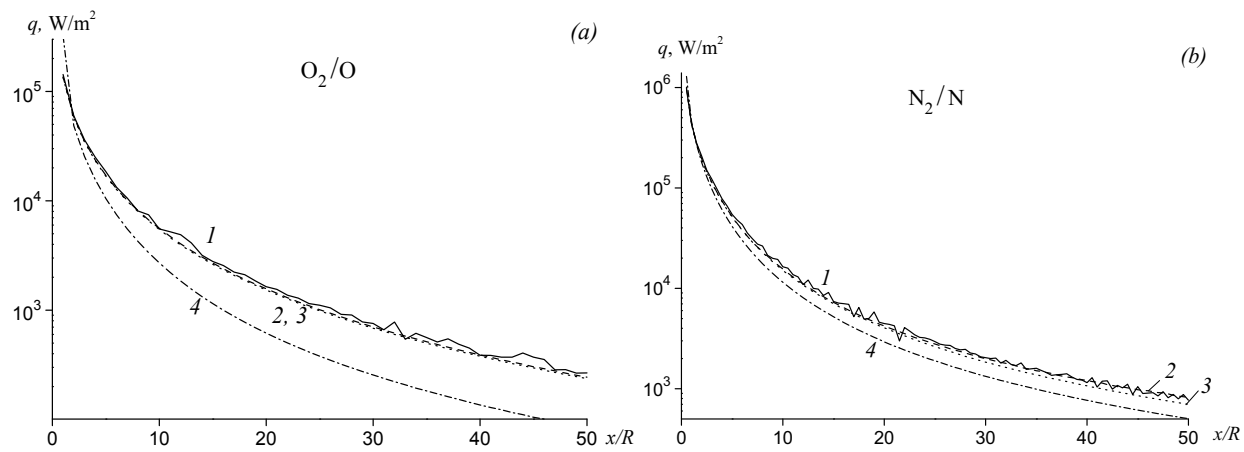

Fig. 4. The heat flux $q$ as a function of $x / R$ in different approaches. The curve 1 represents the state-to-state model; 2 the two-temperature approach for anharmonic oscillators; 3 the two-temperature approach for harmonic oscillators; 4 the one temperature model.

It is explained by the fact that the vibrational distributions behind the shock front calculated within the state-to-state and quasi-stationary models differ substantially already at low levels. Therefore the role of diffusion of vibrationally excited molecules (see section 2.1) in the heat transfer becomes important. On the contrary, in a nozzle flow, at low levels difference between state-to-state and quasi-stationary distributions is negligible and becomes significant at intermediate and high levels. As shown in Kustova, Nagnibeda, Alexandrova \& Chikhaoui (2002), contribution to the heat flux mainly is due to low vibrational levels, therefore difference between heat flux values obtained in different approaches occurs small.

Thereby, to calculate the macroscopic parameters and to estimate the heat transfer in nozzle flows, the multi-temperature approach providing a satisfactory accuracy can be used. Nevertheless, to simulate the non-equilibrium distributions, the more detailed and rigorous state-to-state model should be used.

The same conclusion was obtained for expanding flows of a 5-component air mixture in nozzles of different shapes Capitelli et al. (2002) where the role of the diffusion of vibrational energy was also found to be weak. In Fig. 5a, the effect of the nozzle geometry on the heat flux is shown. Two nozzle shapes are considered: the ONERA F4 nozzle and the parabolic one. The energy flux in the F4 nozzle has a more sharp peak in the vicinity of the throat $(x=0.5 \mathrm{~m})$, its value exceeds that of the heat flux in the parabolic nozzle even at lower initial temperature. It means that the gradients of vibrational level populations and macroscopic parameters in the parabolic nozzle are noticeably less compared to those in the F4 nozzle. Fig. $5 \mathrm{~b}$ represents the total heat flux in the parabolic nozzle at different initial conditions. One can see that $q$ increases significantly with the reservoir temperature $T_{0}$.

The influence of different kinetic models on the heat transfer in a non-equilibrium boundary layer and contribution of different kinetic processes to the total energy transfer are studied for $\mathrm{N}_{2} / \mathrm{N}$ and $\mathrm{O}_{2} / \mathrm{O}$ mixtures in Armenise et al. (2006; 1999); Kustova, Nagnibeda, Armenise \& Capitelli (2002). It is interesting to emphasize noticeable 

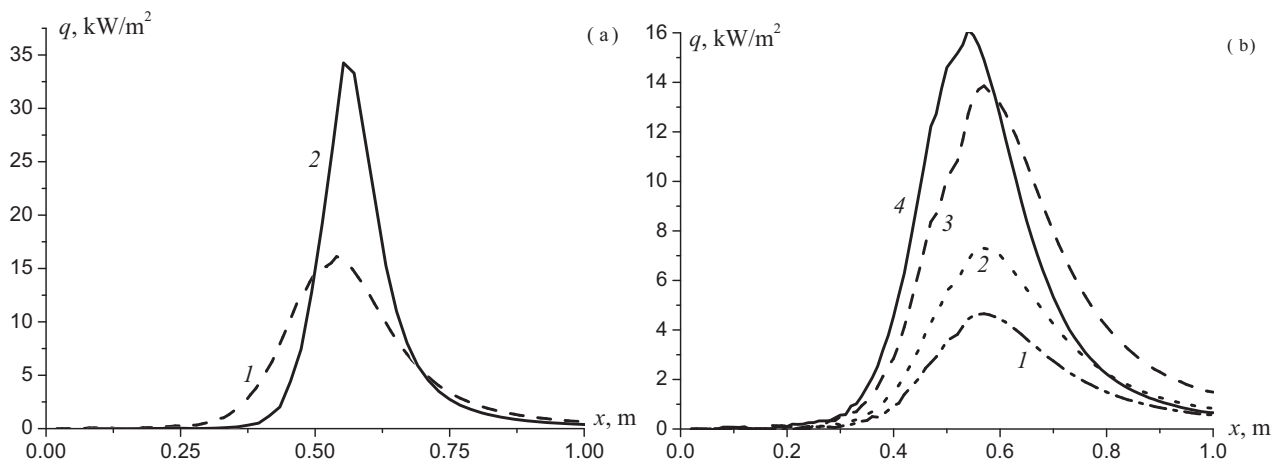

Fig. 5. Total heat flux $q$ as a function of $x$. (a) Various nozzle shapes. The curve 1 represents the parabolic nozzle, $T_{0}=8000 \mathrm{~K}, p_{0}=1.91 \mathrm{~atm} ; 2$ : F4 nozzle, $T_{0}=7000 \mathrm{~K}, p_{0}=300 \mathrm{~atm}$. (b) Parabolic nozzle. The curve 1: $T_{0}=4000 \mathrm{~K}, p_{0}=1.17 \mathrm{~atm} ; 2: T_{0}=5000 \mathrm{~K}, p_{0}=1.21 \mathrm{~atm} ; 3$ : $T_{0}=6000 \mathrm{~K}, p_{0}=1.35 \mathrm{~atm} ; 4: T_{0}=8000 \mathrm{~K}, p_{0}=1.91 \mathrm{~atm}$.

competition of the thermal and mass diffusion processes found for $\mathrm{N}_{2} / \mathrm{N}$ mixture near non-catalytic surface. It is commonly assumed that the effect of thermal diffusion in the boundary layer can be neglected since the thermal diffusion coefficients are small. However, the estimates show that close to the wall the thermal diffusion plays an important role in the heat transfer, reducing the heat flux by the factor of two- to three. The significant contribution of thermal diffusion (despite the small values for the thermal diffusion coefficients) is explained by the high temperature gradient near the surface. The mass diffusion tends to increase the heat flux, its contribution rises from 1\% close to the wall up to $60-65 \%$ at the external edge of the boundary layer. The important effect of recombination in a flow mainly for the diffusive part of the heat flux is found.

The comparison of the heat flux calculated using the non-equilibrium state-to-state distributions with that obtained on the basis of the one-temperature Boltzmann distribution shows the maximum discrepancy is about $18-20 \%$ close to the surface.

Study of heat transfer in $\mathrm{O}_{2} / \mathrm{O}$ mixture flow near the catalytic wall shows an important role of surface catalysis on diffusion processes. On the contrary, for the non-catalytic surface the total heat flux is approximately equal to the Fourier flux associated to the thermal conductivity which indicates a minor contribution of diffusion processes to the heat transfer (in contrast to that in the previous case of $\mathrm{N}_{2} / \mathrm{N}$ mixture for which the role of diffusion was noticeable even for the non-catalytic surface). Also in contrast to the results obtained for $\mathrm{N}_{2} / \mathrm{N}$ mixture, in $\mathrm{O}_{2} / \mathrm{O}$ flow near catalytic surface the effect of thermal diffusion occurs rather insignificant. The contribution of mass diffusion of atoms also appears small, the main role in the total energy transfer belongs to the processes of heat conduction and diffusion of vibrational energy. Near the wall these processes strongly compete.

It should be pointed out that the results concerning heat transfer in a boundary layer discussed above are obtained using the simplified sets of governing equations for viscous gas with constant Prandtl and Schmidt numbers. Then, macroscopic parameters values found from these equations were used in rigorous kinetic theory transport algorithms. Such an approach is not completely self-consistent, since the flow parameters and transport terms remain uncoupled, but it makes possible to take into account the influence of the state-to-state kinetics 
on the flow parameters and heat transfer near the wall. Recently, a similar $\mathrm{N}_{2} / \mathrm{N}$ flow was studied in Orsini et al. (2008) within a more rigorous model incorporating the accurate kinetic theory transport algorithms directly into the boundary layer equations. In this paper, the accurate set of the fluid dynamic equations was solved, and the results are compared to those obtained within the simplified formulation of the problem. Thereby, for the first time, the state-to-state kinetics and transport properties were coupled self-consistently, and the role of the detailed vibrational kinetics in the heat transfer was estimated.

In the paper Kustova et al. (2009), the accurate transport kinetic theory algorithms for multi-temperature mixtures containing $\mathrm{CO}_{2}$ molecules developed in Kustova \& Nagnibeda (2006) in the first time, were implemented directly into the CFD numerical simulations of $2 \mathrm{D}$ viscous hypersonic shock layer near a space craft entering to the Mars atmosphere and the influence of non-equilibrium kinetics on fluid dynamic parameters and heat transfer in a shock layer was shown.

\section{Conclusion}

In the present Chapter, the problem of modelling of heat transfer in high temperature hypersonic flows of reacting gas mixtures is considered. Theoretical models for transport properties in reacting flows are proposed for the conditions of weak and strong deviations from the local thermal and chemical equilibrium. Peculiarities of the kinetic theory transport algorithms developed for various non-equilibrium conditions are discussed and recommendations concerning the choice of an adequate kinetic model for the total energy transfer under particular conditions are given. The results of applications of proposed models for different flows of reacting mixtures are demonstrated.

\section{Acknowledgements}

We are grateful to the Russian Foundation for Basic Research (Grant 11-01-00408) and Ministry of Education and Science of RF (Contract 13.G25.31.0076) for the support of this study.

\section{References}

Adamovich, I. \& Rich, J. (1998). Three-dimensional nonperturbative analytic model of vibrational energy transfer in atom-molecule collisions, J. Chem. Phys. 109(18): 7711-7724.

Armenise, I., Barbato, M., Capitelli, M. \& Kustova, E. (2006). State-to-state catalytic models, kinetics and transport in hypersonic boundary layers, J. Thermophys. Heat Transfer 20(3): 465-476.

Armenise, I., Capitelli, M., Colonna, G. \& Gorse, C. (1996). Nonequilibrium vibrational kinetics in the boundary layer of re-entering bodies, J. Thermophys. Heat Transfer 10(3): 397-405.

Armenise, I., Capitelli, M., Kustova, E. \& Nagnibeda, E. (1999). The influence of nonequilibrium kinetics on the heat transfer and diffusion near re-entering body, $J$. Thermophys. Heat Transfer 13(2): 210-218.

Billing, G. \& Fisher, E. (1979). VV and VT rate coefficients in $N_{2}$ by a quantum-classical model, Chem. Phys. 43: 395-401. 
Brokaw, R. (1960). Thermal conductivity of gas mixtures in chemical equilibrium II, J. Chem. Phys. 32: 1005

Butler, J. \& Brokaw, R. (1957). Thermal conductivity of gas mixtures in chemical equilibrium, J. Chem. Phys. 26: 1636.

Capitelli, M., Colonna, G., Kustova, E. \& Nagnibeda, E. (2002). State-to-state kinetics and transport properties in supersonic air nozzle flows, Proceedings of the 4th European Symposium on Aerothermodynamics for Space Vehicles, ESTEC, Noordwijk, The Netherlands, ESA Publication Division, ESA SP-487. P. 137-143.

Chapman, S. \& Cowling, T. (1970). The Mathematical Theory of Non-Uniform Gases, 3d edn, Cambridge University Press, Cambridge.

Chikhaoui, A., Dudon, J., Genieys, S., Kustova, E. \& Nagnibeda, E. (2000). Multi-temperature kinetic model for heat transfer in reacting gas mixture, Phys. Fluids 12(1): 220-232.

Chikhaoui, A., Dudon, J., Kustova, E. \& Nagnibeda, E. (1997). Transport properties in reacting mixture of polyatomic gases, Physica A 247(1-4): 526-552.

Ern, A. \& Giovangigli, V. (1994). Multicomponent Transport Algorithms, Lect. Notes Phys., Series monographs, M24, Springer-Verlag.

Ern, A. \& Giovangigli, V. (1998). The kinetic chemical equilibrium regime, Physica A 260: 49-72.

Esposito, F., Capitelli, M. \& Gorse, C. (2000). Quasi-classical dynamics and vibrational kinetics in $\mathrm{N}_{2}(v)-N$ system, Chem. Phys. 257: 193-202.

Eucken, E. (1913). Ueber das Wärmeleitvermogen, die Spezifische Wärme und die innere Reibung der Gase, Physik. Zeitschr 14: 324-332.

Ferziger, J. \& Kaper, H. (1972). Mathematical Theory of Transport Processes in Gases, North-Holland, Amsterdam, London.

Gordiets, B. \& Mamedov, S. (1974). Distribution function and vibrational energy relaxation rate in a system of anharmonic oscillators, Prikl. Mekh. Tekhn. Phys. 3: 13-22.

Gordietz, B. \& Zhdanok, S. (1986). Analytical theory of vibrational kinetics of anharmonic oscillators, in M. Capitelli (ed.), Nonequilibrium Vibrational Kinetics, Springer-Verlag, Berlin, Heidelberg, New York, Tokyo, pp. 43-84.

Hirschfelder, J., Curtiss, C. \& Bird, R. (1954). The Molecular Theory of Gases and Liquids, J.Wiley and Sons, New York.

Kustova, E. (2001). On the simplified state-to-state transport coefficients, Chem. Phys. 270(1): 177-195.

Kustova, E. \& Nagnibeda, E. (1997). Vibrational kinetics and transport processes in a strongly non-equilibrium gas, Izvestiya RAN, Mekhanika Zhidkosti i Gasa 5: 150-160.

Kustova, E. \& Nagnibeda, E. (1998). Transport properties of a reacting gas mixture with strong vibrational and chemical nonequilibrium, Chem. Phys. 233: 57-75.

Kustova, E. \& Nagnibeda, E. (1999). State-to-state approach in the transport kinetic theory, Rarefied Gas Dynamics 21, Vol. 1, CEPADUES, Toulouse, France, pp. 231-238.

Kustova, E. \& Nagnibeda, E. (2006). On a correct description of a multi-temperature dissociating $\mathrm{CO}_{2}$ flow, Chem. Phys. 321: 293-310.

Kustova, E., Nagnibeda, E., Alexandrova, T. \& Chikhaoui, A. (2002). On the non-equilibrium kinetics and heat transfer in nozzle flows, Chem. Phys. 276(2): 139-154.

Kustova, E., Nagnibeda, E., Armenise, I. \& Capitelli, M. (2002). Non-equilibrium kinetics and heat transfer in $\mathrm{O}_{2} / \mathrm{O}$ mixtures near catalytic surfaces, J. Thermophys. Heat Transfer 16(2): 238-244. 
Kustova, E., Nagnibeda, E., Shevelev, Y. \& Syzranova, N. (2009). Non-equilibrium supersonic $\mathrm{CO}_{2}$ flows with real gas effects near a blunt body, in T. Abe (ed.), Rarefied Gas Dynamics: 26-th International Symposium, Vol. 1084 of AIP Conference Proceedings, pp. 831-836.

Kustova, E. \& Puzyreva, L. (2009). Transport coefficients in nonequilibrium gas-mixture flows with electronic excitation, Phys. Rev. E 80(4): 046407.

Laganà, A. \& Garcia, E. (1994). Temperature dependence of $N+N_{2}$ rate coefficients, J. Chem. Phys. 98: 502-507.

Nagnibeda, E. \& Kustova, E. (2009). Nonequilibrium Reacting Gas Flows. Kinetic Theory of Transport and Relaxation Processes, Springer Verlag, Berlin, Heidelberg.

Orsini, A., Rini, P., Taviani, V., Fletcher, D., Kustova, E. \& Nagnibeda, E. (2008). State-to-state simulation of non-equilibrium nitrogen stagnation line flows: Fluid dynamics and vibrational kinetics, J. Thermophys. Heat Transfer 22(3): 390-398.

Phys-Chem (2002). Physical and Chemical Processes in Gas Dynamics: Cross Sections and Rate Constants for Physical and Chemical Processes. Volume I, Vol. 196 of Progress in Astronautics and Aeronautics.

Rydalevskaya, M. (1977). Formal kinetic description of gas mixtures with dissociation and recombination, Aerodynamics of Rarefied Gases, Vol. IX, Leningrad University Press, Leningrad, pp. 5-20.

Rydalevskaya, M. (2003). Statistical and Kinetic Models in Physical-Chemical Gas Dynamics, Saint Petersburg Univ. Press, Saint Petersburg.

Stupochenko, Y., Losev, S. \& Osipov, A. (1967). Relaxation in Shock Waves, Springer-Verlag, Berlin, Heidelberg, New York.

Treanor, C., Rich, I. \& Rehm, R. (1968). Vibrational relaxation of anharmonic oscillators with exchange dominated collisions, J. Chem. Phys. 48: 1798.

Vallander, S., Nagnibeda, E. \& Rydalevskaya, M. (1977). Some Questions of the Kinetic Theory of the Chemical Reacting Gas Mixture, Leningrad University Press, Leningrad. Translation: US AirForce FASTC-ID (RS) TO-0608-93.

Wang Chang, C. \& Uhlenbeck, G. (1951). Transport phenomena in polyatomic gases, CM-681, University of Michigan Research Report. 


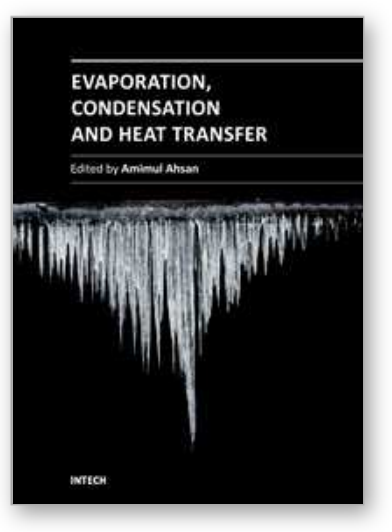

\author{
Evaporation, Condensation and Heat transfer \\ Edited by Dr. Amimul Ahsan
}

ISBN 978-953-307-583-9

Hard cover, 582 pages

Publisher InTech

Published online 12, September, 2011

Published in print edition September, 2011

The theoretical analysis and modeling of heat and mass transfer rates produced in evaporation and condensation processes are significant issues in a design of wide range of industrial processes and devices. This book includes 25 advanced and revised contributions, and it covers mainly (1) evaporation and boiling, (2) condensation and cooling, (3) heat transfer and exchanger, and (4) fluid and flow. The readers of this book will appreciate the current issues of modeling on evaporation, water vapor condensation, heat transfer and exchanger, and on fluid flow in different aspects. The approaches would be applicable in various industrial purposes as well. The advanced idea and information described here will be fruitful for the readers to find a sustainable solution in an industrialized society.

\title{
How to reference
}

In order to correctly reference this scholarly work, feel free to copy and paste the following:

E.V. Kustova and E.A. Nagnibeda (2011). Different Approaches for Modelling of Heat Transfer in NonEquilibrium Reacting Gas Flows, Evaporation, Condensation and Heat transfer, Dr. Amimul Ahsan (Ed.), ISBN: 978-953-307-583-9, InTech, Available from: http://www.intechopen.com/books/evaporation-condensation-andheat-transfer/different-approaches-for-modelling-of-heat-transfer-in-non-equilibrium-reacting-gas-flows

\section{INTECH}

open science | open minds

\section{InTech Europe}

University Campus STeP Ri

Slavka Krautzeka 83/A

51000 Rijeka, Croatia

Phone: +385 (51) 770447

Fax: +385 (51) 686166

www.intechopen.com

\section{InTech China}

Unit 405, Office Block, Hotel Equatorial Shanghai

No.65, Yan An Road (West), Shanghai, 200040, China

中国上海市延安西路65号上海国际贵都大饭店办公楼405单元

Phone: +86-21-62489820

Fax: +86-21-62489821 
(C) 2011 The Author(s). Licensee IntechOpen. This chapter is distributed under the terms of the Creative Commons Attribution-NonCommercialShareAlike-3.0 License, which permits use, distribution and reproduction for non-commercial purposes, provided the original is properly cited and derivative works building on this content are distributed under the same license. 